\title{
A novel method for determining adjacent lung segments with infrared thoracoscopy
}

\author{
Noriyuki Misaki, MD, ${ }^{\text {a }}$ Sung Soo Chang, $\mathrm{MD},{ }^{\mathrm{a}}$ Masashi Gotoh, $\mathrm{MD}, \mathrm{PhD},{ }^{\mathrm{a}}$ Yasumichi Yamamoto, MD, PhD, ${ }^{\mathrm{a}}$ \\ Katashi Satoh, $\mathrm{MD}, \mathrm{PhD},{ }^{\mathrm{b}}$ and Hiroyasu Yokomise, $\mathrm{MD}, \mathrm{PhD}^{\mathrm{a}}$
}

Objectives: We investigated a new technique for identifying the lung intersegmental line using infrared thoracoscopy with intravenous injection of indocyanine green.

\begin{abstract}
Methods: This was an experimental animal study, and target segments were established preoperatively. Six adult beagle dogs underwent thoracotomy. After the corresponding pulmonary artery of the target segment had been ligated, indocyanine green was administered intravenously during infrared thoracoscopy. The lung was separated into 2 areas, white and blue, according to the blood flow on the monitor. We marked the visceral pleura with electrocautery along the transition zone showing a change in color from blue to white. The experimental lung was removed and subjected to pathologic and radiologic analysis.

Results: After injection of indocyanine green, infrared thoracoscopy showed that the area of normal perfusion changed to blue, whereas the area at which perfusion was absent remained white. The transition zone between colors was distinct, and the blue stain remained visible during the marking procedure. Three-dimensional computed tomographic analysis indicated that the marking separated the target segmental bronchus from the adjacent one. Detailed macroscopic and microscopic study confirmed that the marking corresponded to the intersegmental line.
\end{abstract}

Conclusion: By using infrared thoracoscopy with indocyanine green, it is possible to detect the intersegmental line without inflating the lung.

In recent years, segmentectomy has received renewed interest as a useful procedure for patients with certain early-stage lung cancers. ${ }^{1-3}$ Approaches for segmentectomy vary among institutions, but a number of surgeons have decided on the intersegmental line on the basis of inflating or deflating part of the lung. It is widely known that in the respiratory system collateral air flow occurs through the pores of Kohn, the canals of Lambert, and direct airway anastomoses. When lung inflation is initiated, the air often moves into adjacent pulmonary segments to varying degrees as a result of collateral flow. In patients with emphysema, it is often difficult to determine the intersegmental line precisely. Also, if the surgical view is limited, such as during video-assisted thoracic surgery, the inflated lung can sometimes affect the surgical procedure.

In general, each bronchus is accompanied by a pulmonary artery. Therefore if the area supplied by the dominant pulmonary artery of a target segment is known, segmentectomy can be performed without any influence of collateral air drift. In previous studies we have succeeded in visualizing differ-

\footnotetext{
From the Second Department of Surgery, ${ }^{\text {a }}$ Faculty of Medicine, Kagawa University, Kagawa, Japan, and the Department of Nursing, ${ }^{b}$ Kagawa Prefectural College of Health Sciences, Kagawa, Japan.

Received for publication Aug 7, 2008; revisions received Dec 4, 2008; accepted for publication Jan 4, 2009; available ahead of print March 9, 2009.

Address for reprints: Hiroyasu Yokomise, MD, PhD, Second Department of Surgery,

Faculty of Medicine, Kagawa University, 1750-1 Ikenobe, Miki-cho, Kita-gun,

Kagawa 761-0793, Japan (E-mail: yokomise@kms.ac.jp).

J Thorac Cardiovasc Surg 2009;138:613-8

0022-5223/\$36.00

Copyright (C) 2009 by The American Association for Thoracic Surgery

doi:10.1016/j.jtcvs.2009.01.003
}

ential blood flow in the lung using infrared thoracoscopy (IRT) with injection of indocyanine green (ICG). ${ }^{4-6} \mathrm{We}$ have hypothesized that if the dominant pulmonary artery of a target segment is interrupted, it should be possible to visualize the resulting change in blood distribution by using IRT with ICG. In this study we attempted to identify the transition zone affected by interruption of the dominant pulmonary artery relative to the adjacent area using IRT with ICG and to prove that this corresponded to the intersegmental line by using radiographic and pathologic analysis.

\section{MATERIALS AND METHODS}

The principle of IRT has been reported in detail previously. ${ }^{5}$ In brief, light was supplied from a light source and changed to infrared light of 2 different wavelengths ( 805 and $940 \mathrm{~nm}$ ) by passing it through a filter. The infrared light was used to irradiate the lung through the head of the thoracoscope. The reflected light was received by a camera. The data were converted and displayed in a transducer, and the lung was viewed on a monitor as white (Figure 1, $A$ ). ICG absorbs light at $805 \mathrm{~nm}$, and therefore lung tissue containing ICG reflects light at only $940 \mathrm{~nm}$. Thus the normal perfused lung appears blue, and any perfusion defect remains white (Figure 1, B).

\section{Segmental Anatomy}

The right lung of the dog consists of the cranial, middle, caudal, and accessory lobes. The right cranial and caudal lobes were used in this study. The canine pulmonary segmental bronchi were defined in accordance with those for human subjects. The first dorsal branch is represented as the ramus dorsalis (B2) in the cranial lobe. After B2 branches, the cranial branch is the ramus apicalis (B1), and the ventral branch is the ramus ventralis (B3). In the caudal lobe the first dorsal branch is the ramus superior (B6), and the first ventral branch is the ramus ventrobasalis (B8). The segments (S) and 


\section{Abbreviations and Acronyms \\ $\mathrm{CT}=$ computed tomography \\ $\mathrm{ICG}=$ indocyanine green \\ $\mathrm{IRT}=$ infrared thoracoscopy}

pulmonary arteries (A) were named after each bronchus, such as S2 and A2 corresponding to B2 (Figure 2).

\section{Surgical Procedure}

Six adult beagle dogs (body weight, 9-11 kg; mean body weight, $10.2 \mathrm{~kg}$ ) were anesthetized by means of intramuscular injection of xylazine (Selactal; $5.0 \mathrm{mg} / \mathrm{kg}$; Bayer Ltd, Tokyo, Japan) and ketamine (Ketalar; 125 $\mathrm{mg} / \mathrm{kg}$; Sankyo Co, Ltd, Tokyo, Japan). A Univent endobronchial tube (Fuji Systems Co, Tokyo, Japan) was introduced for bronchoscopy after achievement of inhalational anesthesia with sevoflurane. Thoracotomy was performed during single-lung ventilation. The target segment was decided preoperatively. The dominant pulmonary artery of the target segment was ligated, and then $25 \mathrm{mg}$ of ICG was injected rapidly into a forelimb vein for observation of the lung under infrared light. The appearance of the lung was separated into 2 areas, white and blue, according to the blood flow on the monitor. The transition zone between the 2 color areas was then marked to the visceral pleura by means of electrocautery. All animals were killed with intravenous administration of pentobarbital sodium (Nembutal; $50 \mathrm{mg} / \mathrm{kg}$; Dainippon Sumitomo Pharma Co, Ltd, Osaka, Japan), and pneumonectomy was performed to examine the experimental lung radiologically and pathologically.

\section{Specimen Fixation}

The lung specimens were inflated and fixed with the glycol-formalin technique, as described by Markarian and Dailey. ${ }^{7}$ The fixative fluid, which was a 50\%:25\%:10\%:10\% mixture of polyethyleneglycol 400 (Wako Pure Chemical Industries, Ltd, Osaka, Japan), 95\% ethyl ether (Wako), $37 \%$ formalin (Wako), and distilled water, respectively, was injected through the bronchus, and the entire specimen was immersed in the same mixture for 48 hours. The fixative fluid within the lungs was removed by purging with an air supply for 3 days. The resulting specimens were dry but spongy in texture.

\section{Radiologic Study}

Computed tomography (CT) was performed on the fixed specimen 2 times. The first film was obtained without any modification to grasp the bronchial anatomy in the area of interest (nonpainted CT). The second film was obtained after painting the specimen surface with barium to understand its surface anatomy (painted CT). We reconstructed the CT images in 3 dimensions to correlate the surface anatomy with the bronchial anatomy. After the CT examination, the specimens were cut into slices $1 \mathrm{~mm}$ thick horizontally and then examined with radiography and microscopy in detail.

\section{Microscopic Study}

The internal architecture of the thick-slice specimens on $\mathrm{x}$-ray films was compared with the stereomicroscopic findings. The areas at the periphery of adjacent segments were embedded in paraffin before being cut and stained with hematoxylin and eosin. We checked the relationship between the marking and the detailed structures of the peripheral areas by using optical microscopy.

The study protocol was approved by the Kagawa University Ethics Committee for Animal Research. All of the animal experiments were carried out in accordance with the "Guide for the care and use of laboratory animals" prepared by the Institute of Laboratory Animal Resources and published by the National Institutes of Health (publication no. 86; 23, revised 1985).

\section{Without ICG injection}

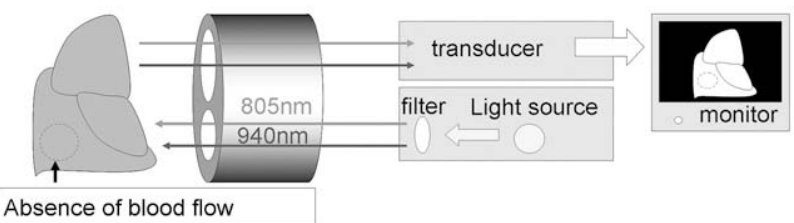

A

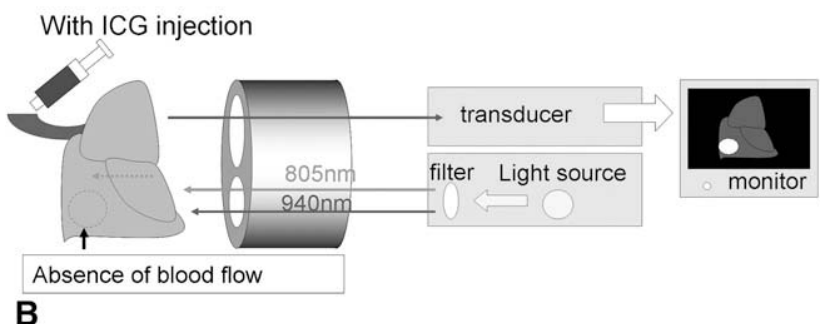

FIGURE 1. The principle of the infrared thoracoscopy system. A, Without indocyanine green $(I C G)$, infrared light at wavelengths of 905 and $805 \mathrm{~nm}$ is reflected, and the object is displayed as white. B, With indocyanine green, the $805-\mathrm{nm}$ light is absorbed, and the object is displayed as blue, whereas the area of the perfusion defect remains white.

\section{RESULTS}

\section{Observation by Normal Light}

We were able to observe the intersegmental line with one of the $\mathrm{S} 1$ models that had an incomplete fissure corresponding to the intended segment (Figure 3, A), and one of the S6 models included a small part of the fissure that is present at the back of the posterior lobe, although the intersegmental line was poorly defined. None of the other models had fissures in the target segments. We were unable to identify the target segments in normal light merely by ligating the dominant pulmonary artery (Figure $3, B$ and $C$ ).

\section{Observation With Infrared Light With or Without ICG}

Before injection of ICG, the lung surfaces appeared under infrared light on the monitor (Figure 3, D-F). As was the

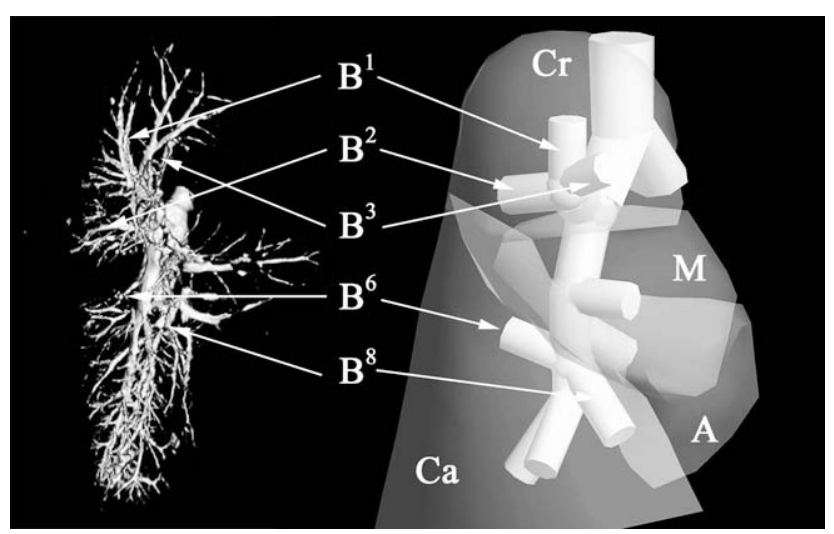

FIGURE 2. Definition of the right lung branches in the dog. $B 1$, Ramus apicalis; $B 2$, ramus dorsalis; $B 3$, ramus ventralis; $B 6$, ramus superior; $B 8$, ramus ventrobasalis; $C r$, Cranial; $M$, middle; $C a$, caudal; $A$, accessory. 

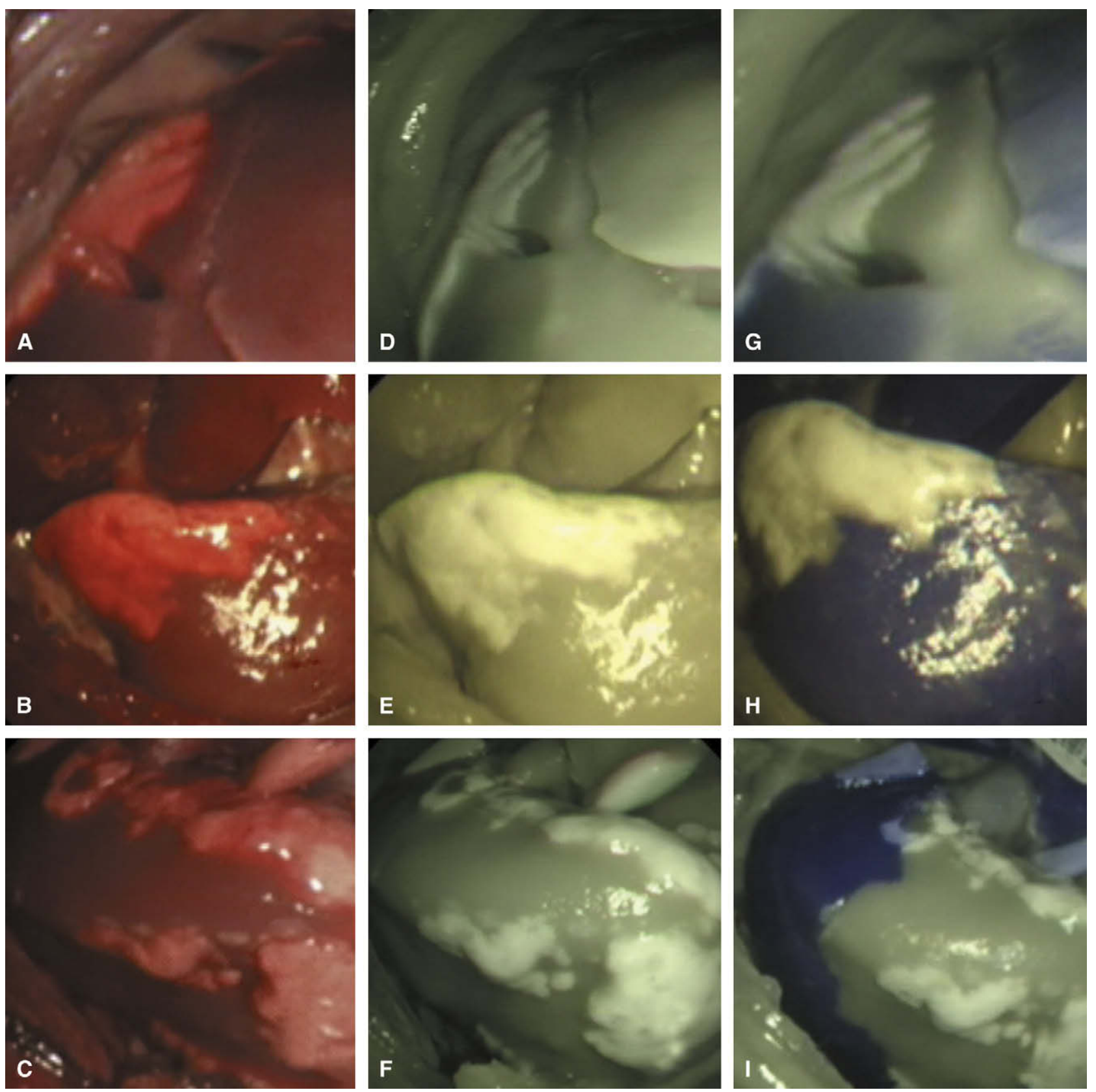

FIGURE 3. The appearance of the lung surface on the monitor (segment $[\mathrm{S}] \mathrm{I}$ : A, D, and G; S6: B, E, and H; and S8: C, F, and I). S1 was isolated in normal light (A), but S6 and S8 were difficult to determine (B and C). The surface color changed to white in infrared light without indocyanine green (ICG; D-F). After injection of indocyanine green, the lung surfaces were separated into white and blue (G-I).

case with normal light, the target segments were unclear without the S1 model. After injection of ICG, the lung surfaces were separated into 2 areas of color, one blue and the other remaining white (Figure 3, G-I). All specimens showed sharp contrast between the white and blue areas and from the hilum to the edge. The $\mathrm{S} 1$ model with a fissure showed contrast at the bottom of the groove (Figure 3, G). The transitional zone, where there was a change in color from blue to white in the visceral pleura, was marked with electrocautery, and the staining remained during the marking. Cases in which there was sufficient information to demonstrate the intersegmental line on the monitor were defined as good, whereas those with insufficient information were defined as poor (Table 1).

\section{Radiographic and Macroscopic Findings}

After fixation of the specimen, the marking of the visceral pleura was retained (Figure 4, A). Nonpainted CT provided information about the bronchial anatomy (Figure 4, $B$ ). The barium coating exactly covered the bronchial anatomy of the 
TABLE 1. Summary of detection of the intersegmental line by means of infrared thoracoscopy

\begin{tabular}{|c|c|c|c|c|c|c|}
\hline \multirow[b]{2}{*}{ Case no. } & \multirow[b]{2}{*}{ Model } & \multirow[b]{2}{*}{ Fissure } & \multicolumn{2}{|c|}{ Detection of intersegmental line } & \multicolumn{2}{|c|}{ Relationship between marking and intersegmental line } \\
\hline & & & Normal light & IRT & 3-D CT & Radiography and microscopy \\
\hline 1 & S1 & + & Good & Good & Matched & Matched \\
\hline 2 & & - & Poor & Good & Matched & Matched \\
\hline 3 & S6 & + & Poor & Good & Matched & Matched \\
\hline 4 & & - & Poor & Good & Matched & Matched \\
\hline 5 & S8 & - & Poor & Good & Matched & Matched \\
\hline 6 & & - & Poor & Good & Matched & Matched \\
\hline
\end{tabular}

$I R T$, Infrared thoracoscopy; 3-D CT, 3-dimensional computed tomography; $S$, segment.

target segment, as seen with painted CT (Figure 4,C). In all specimens the surface anatomy matched the bronchial anatomy in the corresponding area (Table 1).

\section{Microscopic Findings}

The tracings of the x-ray films and stereomicroscopy of the sliced specimens provided detailed information on lung parenchymal structures. The target bronchus and pulmonary artery were separated from other segmental bronchi and arteries by intersegmental structures, such as pulmonary veins, up to the peripheral area, and the intersegmental structures were evident up to the marking of the visceral pleura in all slices. In hematoxylin and eosin-stained specimens, we confirmed the parenchymal structures in relation to the marking, and small branches of bronchi and arteries were divided by the intersegmental veins into each corresponding segment (Figure 5). In all cases the intersegmental structures matched the marking (Table 1). Similar results were obtained in all 6 canine models in which the intersegmental line was detectable by using IRT with ICG.

\section{DISCUSSION}

For many years, general thoracic surgeons have performed segmentectomy for infectious diseases, such as tuberculosis, or benign diseases, such as bronchial ectasia. Several authors have reported the usefulness of anatomic segmentectomy for peripheral small early lung cancers. ${ }^{1-3,8,9}$ Okada and colleagues $^{1}$ reported a mulicenter trial in which sublobar
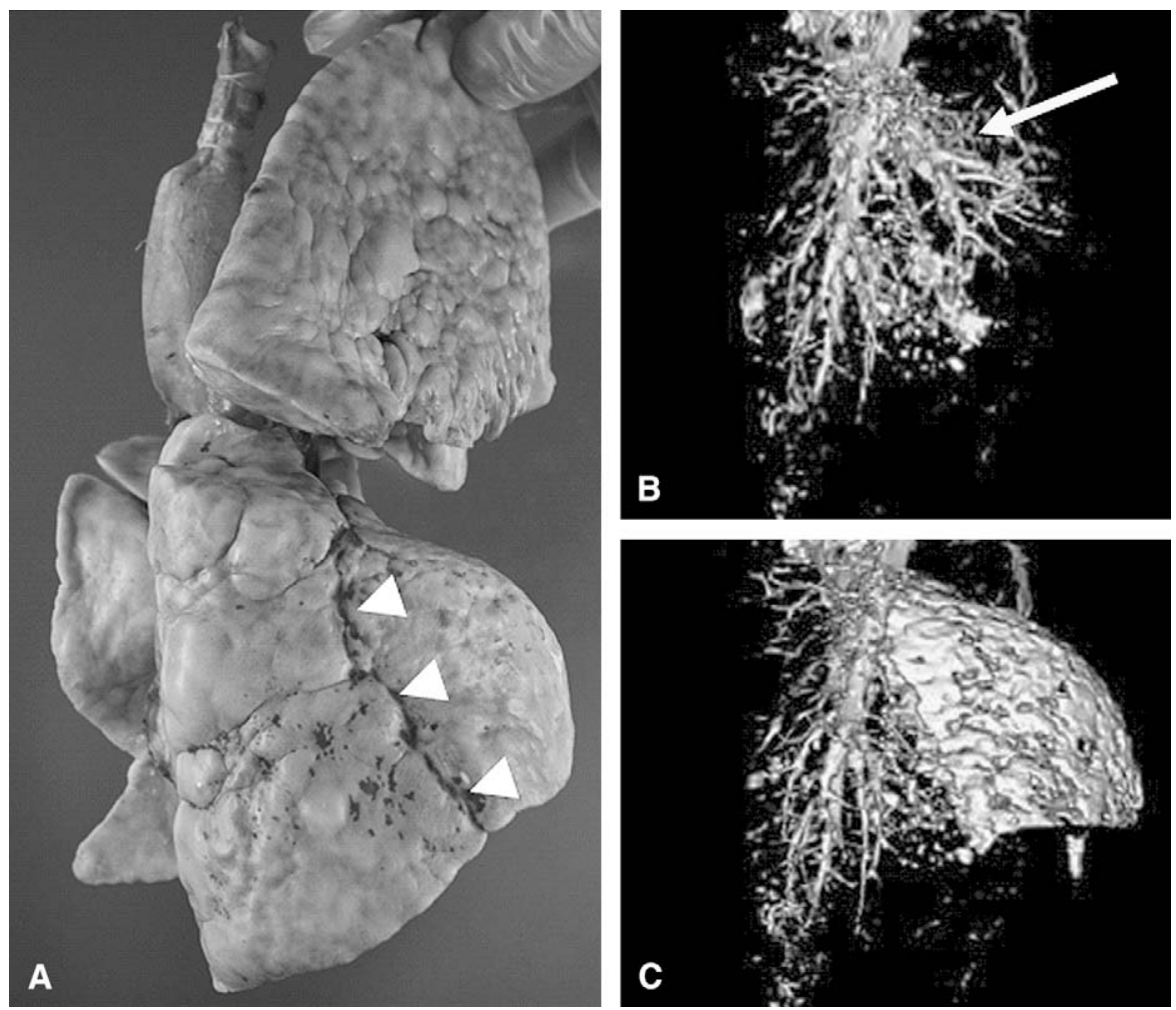

FIGURE 4. An example of the segment (S) 8 model. The arrowhead indicates the macroscopic appearance of the marking of S8 (A). The white arrow indicates B8 in the 3-dimensional image (B). The barium painted on the surface covers the area corresponding to B8 (C). 


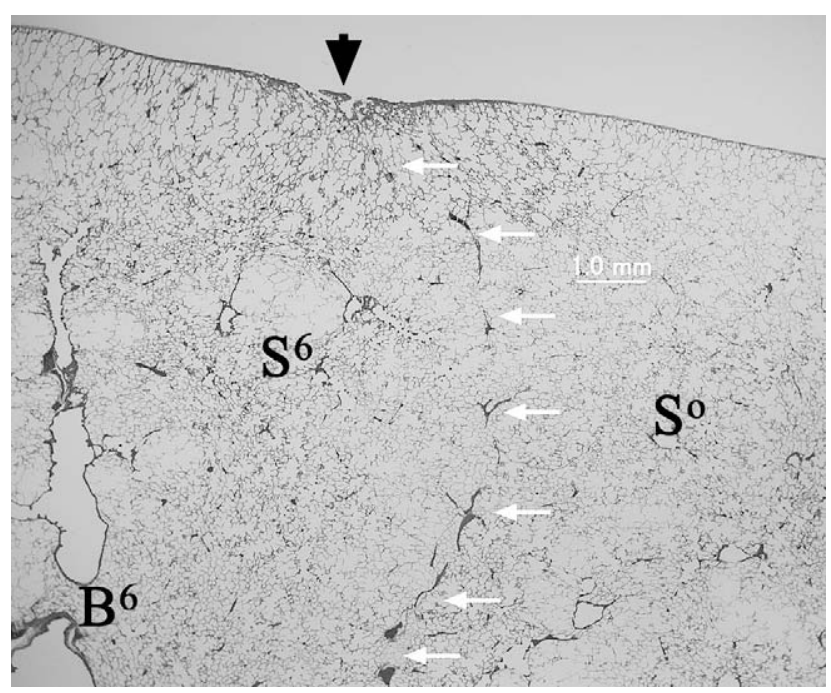

FIGURE 5. Microscopic intersegmental line of segment $(S)$ 6. The marking (black arrow) continues to the intersegmental structure (white arrow), and these structures divide S6 from the other segment (S0). B6 branches were evident in the left corner, and they became terminal branches near the intersegmental line.

resection was compared with lobar resection in patients with early-stage, small-sized non-small cell lung cancers and reported that although both yielded a similar outcome, sublobar resection offered significantly better postoperative lung function. Thus segmentectomy is becoming an important surgical method for some types of lung cancer. With segmentectomy, however, there is a problem of how to identity the intersegmental line. An incomplete intersegmental line can result in congestive lung or ventilatory mismatch. Shuchert and associates ${ }^{9}$ reported that anatomic segmentectomy should certainly be considered in elderly patients and in patients with impaired pulmonary reserve. Elderly patients often have emphysema, and in such cases it is occasionally difficult to identify the intersegmental line when there is collateral air flow or when visualization of the surgical field is limited because of overexpansion of the lung, especially in video-assisted thoracic surgery. It is well known that a pulmonary artery runs together with each bronchus. As a general rule, the segment supplied by the dominant pulmonary artery is the same as that supplied by the dominant bronchus. We have previously reported the use of IRT with ICG intravenous injection to identify emphysematous lesions according to blood supply. ${ }^{4-6}$ IRT with ICG was able to visualize the blood flow of the lung parenchyma on the monitor. In the present study we succeed in visualizing the transition zone in the lung parenchyma after clarifying the dominant pulmonary artery by using IRT with ICG without inflating the lung. This transition zone was confirmed to correspond to the intersegmental line from the hilum to the periphery by means of radiographic and histologic analysis.
The intersegmental line consisted of connective tissues, including the corresponding intersegmental veins of each segment. In clinical segmentectomy for lung cancer, it is necessary to spare the intersegmental veins that drain contiguous segments, consequently preserving the venous drainage of adjacent segments to maintain their full function. However, the surgeon must remove the intersegmental vein if the margin is considered insufficient. ${ }^{9,10}$ It would be useful to know the actual drainage route of the intersegmental veins and the exact distance of the intersegmental veins from the tumor during cancer surgery because this would enable accurate judgment of whether the corresponding intersegmental vein should be incorporated in the resection or whether it should be preserved while leaving an adequate surgical margin during parenchymal division. Sometimes in human subjects part of the target segment is fed by a neighboring segmental small pulmonary artery, and these irregular small vessels exist in the perihilar region. ${ }^{11}$ We were unable to detect such anomalies in the present study, and we considered that they would not have a high degree of clinical relevance because identification of the intersegmental line is required in the peripheral region and the segmental hilar structures become the most reliable landmark of a segment in the perihilar region. Therefore this makes little practical difference when dividing the segmental plain by using the inflation method or the IRT with ICG method.

The main limitation of this study was that we used an animal model and not human subjects, and therefore there is no assurance that the same features would be observed in human subjects. The period of ICG staining is limited, and ICG injection can be done only once. In this study, however, we had enough time to mark the intersegmental line in dogs. We have previously reported that a staining time of 3 to 5 minutes is clinically adequate for detecting emphysematous lesions in human subjects. ${ }^{6}$ There is a possibility that the intersegmental line can be identified not only by the dominant bronchus using the inflating lung method but also by the dominant pulmonary artery using IRT with ICG. Combination of the conventional method with this new method will help to reduce the incidence of ventilation-perfusion mismatch in segmentectomy. Because the present method does not require inflation of the lung, it would be very useful in cases of severe emphysema, in which the intersegmental line cannot be easily identified with the inflation technique.

In conclusion, IRT with ICG makes it possible to identify the target lung segment very easily and quickly without the need for inflation. This method will provide more information for the general thoracic surgeon about intersegmental structure in patients undergoing segmentectomy.

\section{References}

1. Okada M, Koike T, Higashiyama M, Yamato Y, Kodama K, Tubota N. Radical sublobar resection for small-size non-small cell lung cancer: a multicenter study. J Thorac Cardiovasc Surg. 2006;132:769-75. 
2. Keenan RJ, Landreneau RJ, Maley RH Jr, Singh D, Macherey R, Bartley S, et al. Segmental resection spares pulmonary function in patients with stage 1 lung cancer. Ann Thorac Surg. 2004; 78:228-33.

3. Koike T, Yamato Y, Yoshiya K, Shimoyama T, Suzuki R. Intentional limited pulmonary resection for peripheral T1 N0 M0 small-sized lung cancer. J Thorac Cardiovasc Surg. 2003;125:924-8.

4. Gotoh M, Okamoto T, Yamamoto Y, Liu D, Kameyama K, Hayashi E, et al. Development of a canine model of pulmonary emphysema and imaging of the emphysematous lung with infrared thoracoscopy. J Thorac Cardiovasc Surg. 2003;126:1916-21.

5. Gotoh M, Okamoto T, Yamamoto Y, Yokomise H. Real time imaging and quantitative evaluation of the emphysematous lung by infrared thoracoscopy in experimental dogs. ASAIO J. 2005;51:148-51.

6. Gotoh M, Yamamoto Y, Igai H, Chang S, Huang C, Yokomise H. Clinical application of infrared thoracoscopy to detect bullous or emphysematous lesions of the lung. J Thorac Cardiovasc Surg. 2007;134:1498-501.
7. Markarian B, Dailey ET. Preparation of inflated lung specimen. In: Heitzman ER, ed. The lung: radiologic-pathologic correlations. 2nd ed. St Louis: Mosby; 1984, p. 4-12.

8. Schuchert MJ, Pettiford BL, Keeley S, D'Amato TA, Kilic A, Close J, et al. Anatomic segmentectomy in the treatment of stage I non-small cell lung cancer. Ann Thorac Surg. 2007;84:932-3.

9. Schuchert MJ, Pettiford BL, Luketich JD, Landreneau RJ. Parenchymal-sparing resections: why, when, and how. Thorac Surg Clin. 2008;18:93-105.

10. Okada M, Miura T, Ikegaki J, Katoh H, Itoh H, Tsubota N. A novel video-assisted anatomic segmentectomy technique: Selective segmental inflation via bronchofiberoptic jet followed by cautery cutting. J Thorac Cardiovasc Surg. 2007;133: 753-8.

11. Satoh K, Kobayashi T, Mitani M, Kawase Y, Takahashi K, Nishiyama Y, et al. Regular and irregular dichotomies of bronchial branching in the human lung. Acad Radiol. 1996;3:469-74. 